\title{
Microbial and endotoxin emission from composting facilities: characterisation of release and dispersal patterns
}

\author{
L. J. Pankhurst ${ }^{1}$, L. J. Deacon ${ }^{1,2}$, J. Liu ${ }^{3}$, G. H. Drew ${ }^{1}$, \\ E. T. Hayes ${ }^{4}$, S. Jackson ${ }^{3}$, P. J. Longhurst ${ }^{1}$, J. W. S. Longhurst ${ }^{3}$, \\ S. J. T. Pollard ${ }^{1} \&$ S. F. Tyrrel ${ }^{1}$ \\ ${ }^{1}$ School of Applied Sciences, Cranfield University, UK \\ ${ }^{2}$ Environmental Knowledge Transfer Network, UK \\ ${ }^{3}$ Centre for Research in Biomedicine, UWE, Bristol, UK \\ ${ }^{4}$ Air Quality Management Resource Centre, UWE, Bristol, UK
}

\begin{abstract}
The potential risk to human health posed by exposure to bioaerosols released from composting is an important issue. Further growth in the number of composting facilities in the UK is anticipated as biodegradable waste is diverted from landfill. To date, studies of bioaerosol emission from composting have focussed on culturable bioaerosols. This paper describes both culturable bioaerosol and endotoxin release and dispersal from two large green waste composting facilities in the UK. Aspergillus fumigatus, actinomycetes, Gramnegative bacteria, and endotoxins were simultaneously and repeatedly sampled to describe the release and dispersal from these sites. Meteorological and site operational observations were recorded, allowing analysis of factors influencing bioaerosol release and dispersal. The highest measured concentrations of bioaerosols were associated with composting activities such as shredding and turning. Between release and $50-80 \mathrm{~m}$ downwind bioaerosol concentrations reduced by $80-90 \%$. An unexpected second peak was detected 100-150m downwind from source at both sites. Endotoxin dispersal patterns were site specific and showed some differences to dispersal patterns of culturable microorganisms.
\end{abstract}

Keywords: bioaerosol, aspergillus fumigatus, actinomycetes, gram-negative bacteria, endotoxins, composting, dispersal. 


\section{Introduction}

Within the UK, increasing amounts of biodegradable waste are being diverted to composting facilities in order to meet targets set through the Landfill Directive (EC/31/99). In 2006/07 3.6 million tonnes of waste was composted in the UK, with $79 \%$ of this waste processed in open-air turned windrow facilities (The Composting Association [1]). The successful composting of this waste is dependent on a host of thermophilic and thermotolerant microorganisms, whose proliferation is encouraged. However, open-air windrow composting requires several high-energy processes, such as shredding, turning, and screening. While static compost windrows allow the aerosolisation of some composting microorganisms, these agitation processes increase their release dramatically. Past research has shown how activities result in a 3-log increase in bioaerosol emissions (Clark et al [2]; Taha et al [3, 4]).

Bioaerosols can be defined as any aerosol of biological origin (Swan et al [5]). In the case of composting the term refers specifically to microorganisms, their constituent parts, and by-products. Common composting bioaerosols of concern include Aspergillus fumigatus, actinomycetes, and endotoxins (Millner et al [6]). Exposure to these bioaerosols has been shown to result in a range of respiratory conditions, including asthma, mucosal membrane inflammation, and invasive aspergillosis (Dutkiewicz [7]; Swan et al [5]). Tentative, precautionary threshold levels of $1000 \mathrm{CFU} \mathrm{m}^{-3}$ for total bacteria and total fungi, and $300 \mathrm{CFU}$ $\mathrm{m}^{-3}$ for Gram-negative bacteria, have been recommended; furthermore, facilities should preferably not be situated within $250 \mathrm{~m}$ of sensitive receptors (Environment Agency [8]).

Despite the health risks and regulatory limits, the dispersal range of bioaerosols remains uncertain (Albrecht et al [9]). Many past studies present dispersal ranges based on few sampling occasions with limited sampling times (Swan et al [5]) and using mean rather than peak bioaerosol concentrations. In order to produce valid data, the episodic nature of bioaerosol emissions must be accounted for; therefore peak emission data should be enumerated and presented (Albrecht et al [9]). The physical properties of bioaerosols remain poorly characterised (Drew et al [10]; Swan et al [5]), hence behaviour in the atmosphere remains uncertain and atmospheric modelling programs are unable to accurately represent bioaerosol dispersal. In addition, sampling and enumeration methods used for bioaerosols remain underdeveloped compared to those used within soil and aquatic microbiology (Malik et al [11]; Sykes et al [12]). Bioaerosol concentrations may therefore have been consistently underestimated, as the non-culturable fractions remain poorly quantified (Swan et al [5]).

The above factors have contributed to gaps in our understanding of bioaerosol emission and dispersal from composting facilities. It has been suggested that current guidelines surrounding bioaerosols, and their sampling and enumeration, require updating (Environment Agency [13]). A thorough understanding of bioaerosol dispersal is required, given their potential human health impacts. This study enumerates culturable microorganism and endotoxin emission and dispersal from composting facilities through an extensive experimental program; 
creating a validated dispersal profile for these bioaerosols, of use within academia, regulatory bodies, and industry.

\section{Methods}

Two composting facilities in the UK were sampled. Both sites are large-scale green waste composting facilities that receive approximately 25,000 tonnes of waste per annum from civic amenity sites and kerbside collection, and operate open-air turned windrow systems.

Site A is located on a closed and capped landfill facility. Bunding encircles the northern, western and southern sides, with hedgerow on all sides. The nearest residence is located $500 \mathrm{~m}$ to the north of the site. Surrounding land is agricultural, with an area of woodland to the west. Site B is located on the southwest corner of an industrial estate; the northern edge of the site is surrounded by concrete bunding. The industrial estate extends approximately $630 \mathrm{~m}$ to the north and $500 \mathrm{~m}$ to the east of the facility, workplaces can be found $50 \mathrm{~m}$ from the site to the edges of the industrial estate. A large river bounds the west side of the site; arable land surrounds the southern edge of the site and the industrial estate.

SKC personal aerosol filter samplers were used to collect bioaerosols, with a flow rate of $2.2 \pm 0.1 \mathrm{~L} \mathrm{~min}^{-1}$; sampling heads were elevated to $1.7 \mathrm{~m}$, and loaded with sterile polycarbonate filters, pore size $0.8 \mu \mathrm{m}$ (SKC Ltd, UK) (Taha et al [3]). A Kestrel 4000 weather data logger (Nielsen Kellerman, PA, USA) was used to record meteorological conditions throughout sampling.

Filters for culture were placed into buffer solution ( $\mathrm{NaCl} 1 \mathrm{~g} \mathrm{l}^{1}$ and 3 drops of Tween $80 \mathrm{~L}^{-1}$, in 11 sterilised $\mathrm{ddH}_{2} \mathrm{O}$ ) and stored at $4^{\circ} \mathrm{C}$. Within 24 hours samples were processed under aseptic conditions. Samples were shaken to create a suspension, which was diluted to a common logarithm order $\left(10^{-1}\right.$ and $\left.10^{-2}\right)$ and $100 \mu \mathrm{l}$ of each dilution was transferred onto a range of media. Malt Extract Agar (MEA) was used to culture Aspergillus fumigatus, Compost Agar (CA) (Taha et al [4]) was used for actinomycetes, while Gram-negative bacteria were grown using MacConkey Agar (MAC). Plates were incubated at $37 \pm 2{ }^{\circ} \mathrm{C}$ for 3-7 days (MEA and MAC) or $44 \pm 2{ }^{\circ} \mathrm{C}$ for 7 days (CA). Colony forming units (CFUs) were visually enumerated and converted to $\mathrm{CFU} \mathrm{m} \mathrm{m}^{-3}$ of air (Taha et al $[3,4]$ ).

Samples for endotoxin assay were sealed with the cap and clip supplied (IOM multi-dust cassettes) and stored at $-20{ }^{\circ} \mathrm{C}$ until extraction at UWE Bristol. Extraction solution was prepared in Limulus Amebocyte Lysate (LAL) grade water (Lonza Wokingham Limited, UK) containing $0.05 \%$ Tween 20. The extraction solution was centrifuged at $1000 \times \mathrm{g}$ for 15 minutes, the supernatant was collected, vortexed and aliquots placed into two pyrogen-free glass tubes. Analysis was carried out using the PyroGene rFC Endotoxin detection kit (Lonza Wokingham Limited, UK) (Spaan et al [14]).

At Site A, a total of 17 sampling trips were made, from September 2007 until August 2008. Sampling at Site B commenced in August 2008, and is ongoing with 10 sampling trips reported. Samples were taken from on-site, to site boundary (edge of the composting area), and the farthest possible point downwind (Site A: 355m, Site B: 600m). Upwind samples were taken to 
evaluate 'background' concentrations. Samples were taken regardless of weather conditions or site activities.

\section{Results}

\subsection{Aspergillus fumigatus}

A. fumigatus concentrations and dispersal patterns show many similarities at Sites A and B (Figures 1-2). Downwind concentrations are characterised by a significant increase of 1-2 log from upwind to those found on-site during composting activities. Between this peak in concentration and 50-80 m downwind, concentrations decline by approximately $90 \%$. However, a second peak in concentration can be observed $100 \mathrm{~m}$ downwind. From this point a steady decline in A. fumigatus concentrations can be observed. In the case of Site B, a third peak in concentration is visible at 500-600m downwind.

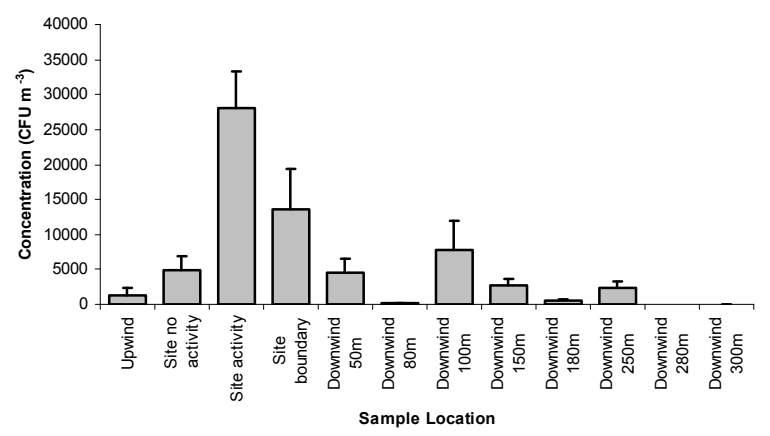

Figure 1: Mean Site A A. fumigatus concentrations presented in CFU m${ }^{-3}$ represented by bar, standard error represented by whisker.

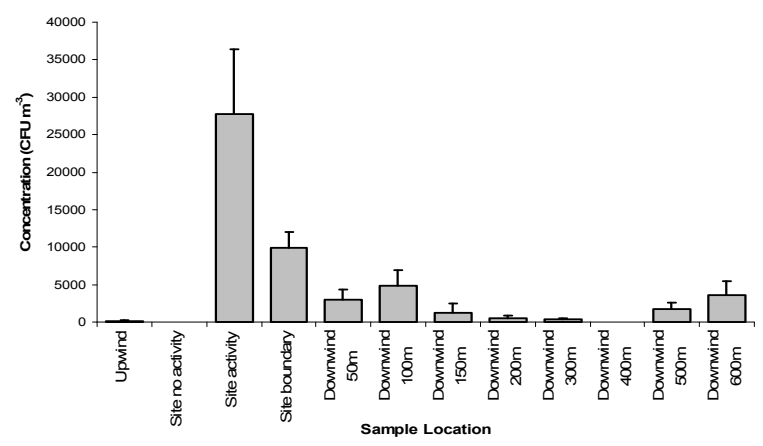

Figure 2: Mean Site B A. fumigatus concentrations presented in CFU m${ }^{-3}$ represented by bar, standard error represented by whisker. 


\subsection{Actinomycetes}

There are some site specific features for actinomycete release and dispersal patterns (Figures 3-4). For Site B, the highest concentrations are found at the site boundary, where they are 2-log above those found upwind and 1-log higher than the Site A peak, which was found on-site during activities. At both sites, concentrations are significantly above those found upwind at the on-site activity, site boundary, and downwind $100-150 \mathrm{~m}$ locations. At Site A there is no significant difference between the concentrations found at any other sampling location and upwind. At Site B, a third peak in concentrations is shown 500$600 \mathrm{~m}$ downwind, with concentrations significantly above those found upwind.

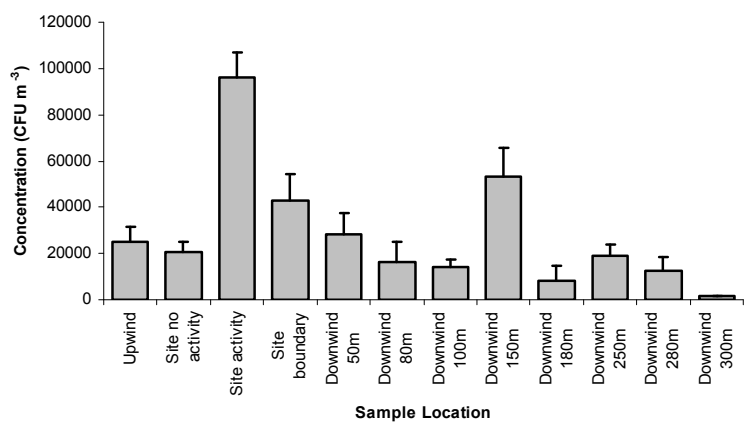

Figure 3: Mean Site A actinomycete concentrations presented in CFU $\mathrm{m}^{-3}$ represented by bar, standard error represented by whisker.

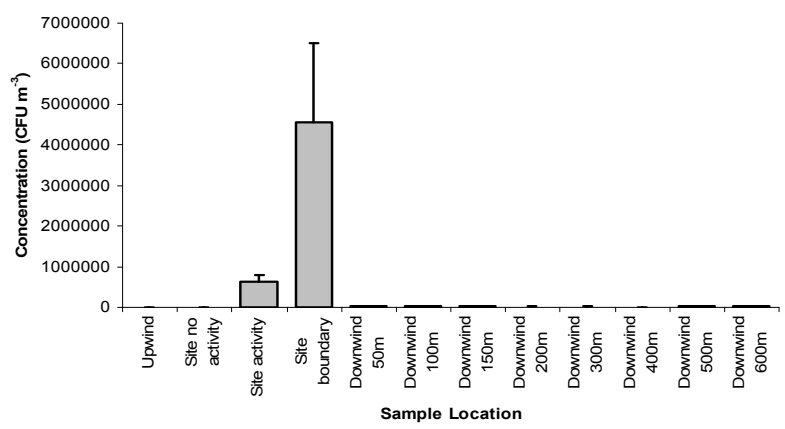

Figure 4: Mean Site B actinomycetes concentrations presented in CFU $\mathrm{m}^{-3}$ represented by bar, standard error represented by whisker. 


\subsection{Gram-negative bacteria}

Gram-negative bacteria were found in the highest concentrations on-site during activity at Site A, and at the site boundary of Site B (Figures 5-6). These concentrations are up to 3-log higher than those found upwind. Downwind concentrations are characterised by a decline of over $90 \%$ between the peak and 50-80m downwind. At both sites a second peak, significantly different from concentrations found upwind, is seen at $150 \mathrm{~m}$ downwind. This peak is more significant at Site A; nevertheless, concentrations reached those found upwind by $280 \mathrm{~m}$ downwind. At Site B, concentrations do not decline to upwind concentrations by $600 \mathrm{~m}$ from site boundaries.

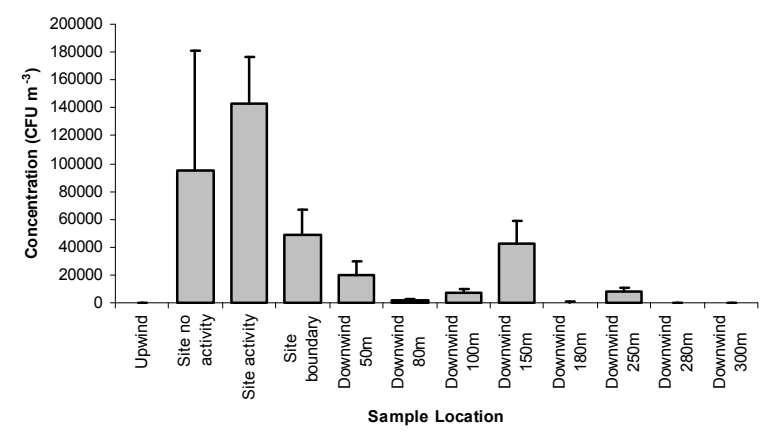

Figure 5: Mean Site A Gram-negative bacteria concentrations presented in CFU $\mathrm{m}^{-3}$ represented by bar, standard error represented by whisker.

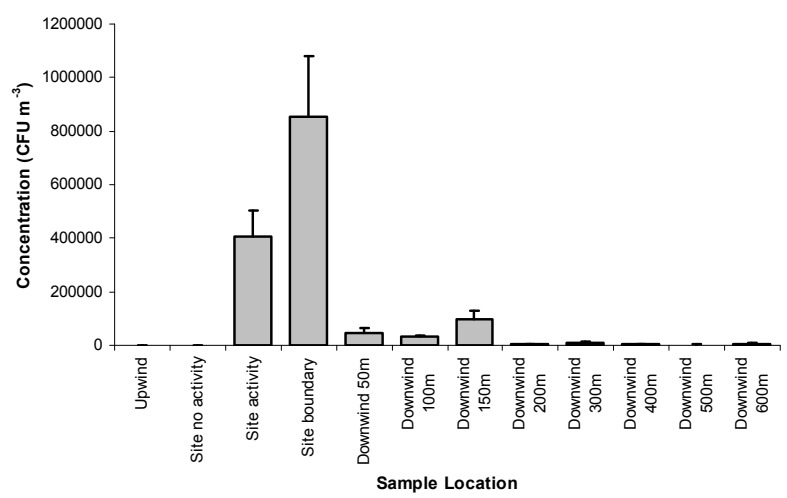

Figure 6: Mean Site B Gram-negative bacteria concentrations presented in CFU $\mathrm{m}^{-3}$ represented by bar, standard error represented by whisker. 


\subsection{Endotoxin}

At both sites, a peak in endotoxin concentrations was found at the site boundary, with concentrations from on-site to downwind $280 \mathrm{~m}$ at Site A, and $200 \mathrm{~m}$ at Site $\mathrm{B}$ above those found upwind (Figures 7-8). Downwind concentrations at both sites are characterised by a decline of $90 \%$ from site boundary to $50 \mathrm{~m}$ downwind. A second peak can be seen at $150 \mathrm{~m}$ downwind for Site A, and $200 \mathrm{~m}$ downwind for Site B. However, at Site A endotoxins showed the highest concentrations $280 \mathrm{~m}$ from site boundary, While at Site B by $300 \mathrm{~m}$ downwind concentrations had returned to those found upwind.

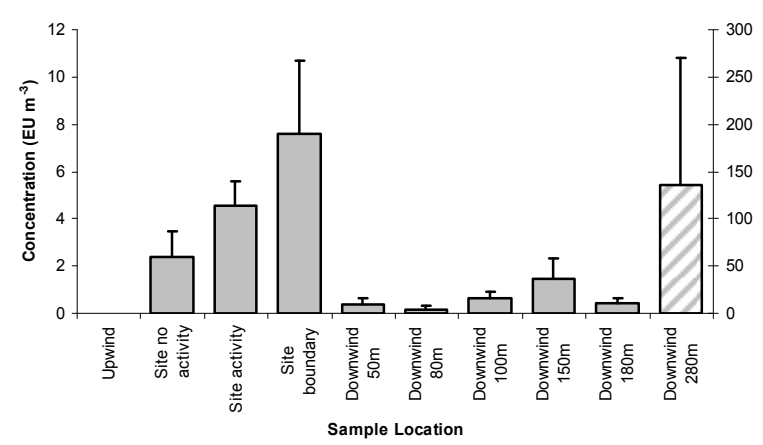

Figure 7: Mean Site A endotoxin concentrations presented in endotoxin units per cubed metre $\left(\mathrm{EU} \mathrm{m}^{-3}\right)$ represented by bar, standard error represented by whisker. Diagonally shaded bar represented on secondary y-axis.

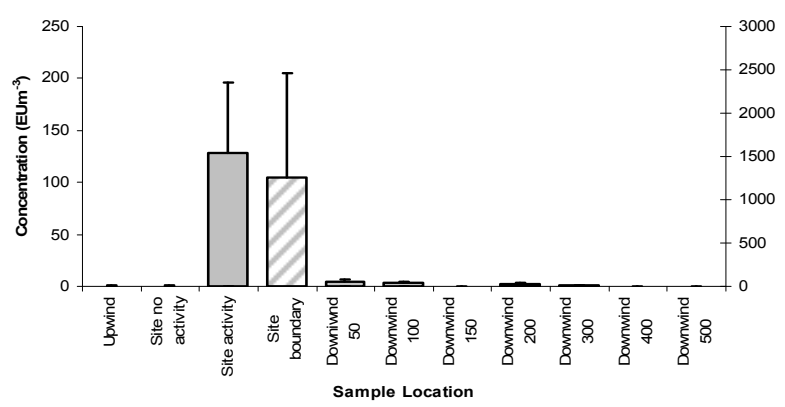

Figure 8: $\quad$ Mean Site B endotoxin concentrations presented in endotoxin units per cubed metre $\left(E U \mathrm{~m}^{-3}\right)$ represented by bar, standard error represented by whisker. Diagonally shaded bar represented on secondary y-axis. 


\section{Discussion}

All of the bioaerosols enumerated through culture showed similar patterns of release; characterised by peak emissions on-site (including site boundary). This is in agreement with Albrecht et al [9] and Taha et al [3]. At Site B, the highest concentrations of actinomycetes and Gram-negative bacteria were found at site boundaries. This may be due to an accumulation of bacteria here as several activities are carried out upwind. Dispersal patterns were also similar, culturable microorganisms showed an $80-90 \%$ reduction in concentrations between peak concentrations and 50-80m downwind. This feature of dispersal has previously been reported anecdotally (Environment Agency [15]). Between 100 and 150m downwind a second peak in concentrations was detectable; this may be due to buoyancy effects, causing some bioaerosols to rise above sampling height until cooled enough to sink back to sampling height. This pattern of dispersal was shown by both sites, suggesting that it is not due to site-specific features.

A. fumigatus reached concentrations similar to those found upwind, and below $1000 \mathrm{CFU} \mathrm{m} \mathrm{m}^{-3}$, by $280 \mathrm{~m}$ from Site A, and $200 \mathrm{~m}$ from Site B. At Site B, however, A. fumigatus concentrations show a further peak $500-600 \mathrm{~m}$ from site boundaries. It is currently unknown whether this peak is due to site emissions or a secondary source. Actinomycetes reached concentrations similar to those found upwind by $180 \mathrm{~m}$ from Site A, and $300 \mathrm{~m}$ from Site B. At both sites, actinomycetes were found in concentrations above the $1000 \mathrm{CFU} \mathrm{m}^{-3}$ limit for total bacteria, even at upwind locations. This may have implications for the future revision of threshold levels, as the relatively high background concentrations of actinomycetes needs to be accounted for. Gram-negative bacteria declined to upwind concentrations, and were below $300 \mathrm{CFU} \mathrm{m}^{-3}$, by $280 \mathrm{~m}$ from Site A. At Site B, concentrations did not decline to those found upwind at any sampling location.

While $A$. fumigatus was released in similar quantities at both sites; this was not the case with the bacteria measured. Actinomycetes peaked at quantities 1$\log$ higher at Site B than Site A. However, up- and downwind concentrations were largely in the same order of magnitude at both sites. Site B Gram-negative bacteria also peaked at concentrations 1-log above those found at Site A. As with actinomycetes, other sampling locations showed concentrations largely within the same order of magnitude at both sites. The higher quantities of bacteria found at Site B may be due to differences in feedstock, as it receives more domestic biodegradable waste than Site A. Furthermore, concentrations at site boundary at Site B are typically higher than at Site A. This site-specific feature may be due to site design, and warrants further investigation. Despite these differences in concentration, dispersal patterns off-site remain similar at both sites.

Endotoxin dispersal patterns show several differences. At both sites, peak onsite concentrations are seen at the site boundary and are similar to those previously found (Liebers et al [16]). As with culturable microorganisms, this peak is followed by a $90 \%$ decline in concentrations. At Site B, this decline is followed by a further small peak at $200 \mathrm{~m}$ downwind, after which concentrations are similar to those found upwind, or undetectable. At Site A, a second peak in 
concentration is seen at $150 \mathrm{~m}$ downwind, as with culturable microorganisms. However, the highest peak was seen at $280 \mathrm{~m}$ downwind, where concentrations were 2-log higher than those found on-site. This feature was not seen at Site B, despite the fact that emissions were 3-log higher on-site as compared to Site A.

\section{Conclusions}

This data-set demonstrates how bioaerosols do not follow a typical decay curve from emission to background concentrations. Peaks in concentration at 100 $150 \mathrm{~m}$ downwind suggest that buoyancy and air temperature impact upon their dispersal. The initial $80-90 \%$ decay in concentrations, followed by a small peak, appears to be a common feature of bioaerosol dispersal, despite the volume of bioaerosols released. This feature of dispersal was previously unquantified. However, the bioaerosols measured also showed some differences in dispersal. Actinomycete concentrations should be interpreted with care, as they were found above reference values at most locations. Furthermore, although Gram-negative bacteria reached background concentrations at Site A, they did not at Site B and remained above the threshold limit.

Bioaerosols may be found above threshold values at distances above the risk assessment limit of $250 \mathrm{~m}$, where sensitive receptors can be exposed, and at upwind locations. This suggests that caution is required in the interpretation of results, and that samples must be repeated in order to ensure valid results.

The creation of this data-set has provided validated dispersion profiles for these bioaerosols, and demonstrated how physical properties affect dispersal range. Further data collection and detailed analysis is continuing in order to add to the data-set. The information provided can be used to improve best-practice risk assessment of bioaerosols, as well as provide a basis for further investigations into bioaerosol dispersal.

\section{Acknowledgements}

The authors would like to acknowledge UWE Bristol for their analysis of samples for endotoxin content. The financial support of SITA part of the SUEZ Environment group and NERC (NERC grant NE/E008534/1) is also acknowledged.

\section{References}

[1] The Composting Association, The State of Composting and Biological Waste Treatment in the UK 2007/07, pp 4, UK, 2008.

[2] Clark, C. S., Rylander, R. and Larsson, L., Levels of Gram-negative bacteria, Aspergillus fumigatus, dust, and endotoxin at compost plants, Applied and Environmental Microbiology, 45 (5), pp. 1501-1505, 1983.

[3] Taha, M. P. M., Drew, G. H., Longhurst, P. J., Smith, R. and Pollard, S. J. T., Bioaerosol releases from compost facilities: Evaluating passive and 
active source terms at a green waste facility for improved risk assessments, Atmospheric Environment, 40, pp. 1159-1169, 2006.

[4] Taha, M. P. M., Drew, G. H., Tamer-Vestlund, A., Aldred, D., Longhurst, P. J. and Pollard, S. J. T., Enumerating actinomycetes in compost bioaerosols at source - Use of soil compost agar to address plate 'masking', Atmospheric Environment, 41, pp. 4759-4765, 2007.

[5] Swan, J. R. M., Kelsey, A., Crook, B., and Gilbert, E.J., Occupational and Environmental Exposure to Bioaerosols from Composts and Potential Health Effects - A critical review of published data, Health and Safety Executive, UK, 2003.

[6] Millner, P. D., Olenchock, S. A., Epstein, E., Rylander, R., M.D., Haines, J., Walker, J., Ooi, B. L., Horne, E. and Maritato, M., Bioaerosols associated with composting facilities, Compost Science and Utilization, 2 (4), pp. 6-57, 1994.

[7] Dutkiewicz, J., Bacteria and fungi in organic dust as potential health hazard, Annals of Agriculture and Environmental Medicine, 4 (1), pp. 11 16, 1997.

[8] Environment Agency, Health Effects of Composting. A study of three compost sites and review of past data, Bristol, UK, 2001.

[9] Albrecht, A., Fischer, G., Brunnemann-Stubbe, G., Jäckel, U. and Kämpfer, P. Recommendations for study design and sampling strategies for airborne microorganisms, MVOC and odours in the surrounding of composting facilities. International Journal of Hygiene and Environmental Health, 211, pp. 121-131, 2008.

[10] Drew, G. H., Tamer, A., Taha, M. P. M., Smith, R., Longhurst, P. J., Kinnersley, R. and Pollard, S. J. T. Dispersion of bioaerosols from composting facilities, Waste 2006 Conference, 19-21st September, Stratford, UK, 2006.

[11] Malik, S., Beer, M., Megharaj, M., and Naidu, R. The use of molecular techniques to characterize the microbial communities in contaminated soil and water, Environment International, 34, pp. 265 - 276, 2008.

[12] Sykes, P., Jones, K. and Wildsmith, John. D. Managing the potential public health risks from bioaerosol liberation at commercial composting sites in the UK: An analysis of the evidence base. Resources, Conservations and Recycling, 52 (2), pp. 410-424, 2007.

[13] Environment Agency. Updated Review of Methods for Monitoring Compost Bioaerosols. Environment Agency, Bristol, UK, in press.

[14] Spaan, S., Wouters, I. M., Oosting, I., Doekes, G. and Heederik, D., Exposure to inhalable dust and endotoxins in agricultural industries, Journal of Environmental Monitoring, 8, pp. 63-72, 2005.

[15] Environment Agency. Monitoring the Environmental Impact of Waste Composting Plants. R\&D Technical Summary P1-216, Environment Agency, Bristol, UK, 2001.

[16] Liebers, V., Brüning, T. and Raulf-Heimsoth, M., Occupational endotoxin exposure and possible health effects on humans, American Journal of Industrial Medicine, 49, pp. 474-491, 2006. 